\title{
Glottopol
}

Revue de sociolinguistique en ligne

$34 \mid 2020$

Les « langues de France ", 20 ans après

\section{Les langues polynésiennes et kanak, des « langues de France » en contexte de décolonisation}

Jacques Vernaudon

\section{OpenEdition}

\section{Journals}

Édition électronique

URL : https://journals.openedition.org/glottopol/488

DOI : $10.4000 /$ glottopol.488

ISSN : 1769-7425

Éditeur

Presses universitaires de Rouen et du Havre

Référence électronique

Jacques Vernaudon, «Les langues polynésiennes et kanak, des «langues de France » en contexte de décolonisation », Glottopol [En ligne], 34 | 2020, mis en ligne le 01 juillet 2020, consulté le 02 octobre 2021. URL : http://journals.openedition.org/glottopol/488; DOI : https://doi.org/10.4000/glottopol.488

Glottopol 


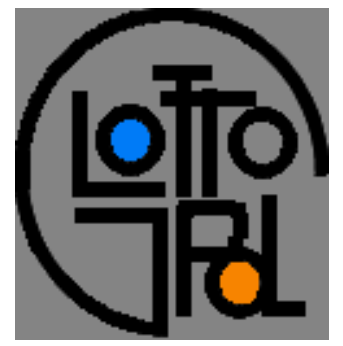

\section{GLOTTOPOL}

Revue de sociolinguistique en ligne $\mathrm{n}^{\circ} 34$ - juillet 2020

Les "langues de France " : 20 ans après

\section{SOMMAIRE}

Hommages à Jean Le Dî

Christian Lagarde : «Langues de France ». Au-delà du symbolique?

Entretien avec Bernard Cerquiglini, par Christian Lagarde.

Entretien avec Paul de Sinety, Délégué général à la langue française et aux langues de France, par Christian Lagarde.

Georg Kremnitz : La problématique initiale de la liste Cerquiglini et ses effets ultérieurs.

Alain Viaut : De "langue régionale » à " langue de France » ou les ombres du territoire.

Wanda Mastor: Le statut constitutionnel des langues régionales en droit comparé. De la reconnaissance à l'indifférence.

Philippe Martel, Marie-Jeanne Verny: Les langues régionales au Parlement, ou l'éternel retour.

Romain Colonna : Les "langues de France » : des langues non-étatiques au pays de l'Étatnation.

Hervé le Bihan : La langue bretonne : une visibilité toute en retenue.

Véronique Bertile : Les langues d'outre-mer: des langues de France? Approche juridique.

Jacques Vernaudon: Les langues polynésiennes et kanak, des "langues de France » en contexte de décolonisation.

Luc Biichlé : Qu'advient-il de l'arabe de France? Mise en perspective sociolinguistique...

Pascal Ottavi : L'épervier, la cage et le passereau.

Marielle Rispail : Le francique lorrain, langue de France? Réflexions et témoignages.

\section{Compte rendu de lecture}

Par Salih Akin : Jean Le Dû \& Yves Le Berre, Métamorphoses. Trente ans de sociolinguistique à Brest (1984-2014), Brest, Centre de Recherche Bretonne, 2019, 302 p. 


\title{
LES LANGUES POLYNÉSIENNES ET KANAK, DES « LANGUES DE FRANCE » EN CONTEXTE DE DÉCOLONISATION
}

\author{
Jacques Vernaudon \\ Université de la Polynésie française, Équipe d'Accueil Sociétés \\ Traditionnelles et Contemporaines en Océanie (EASTCO EA 4241)
}

Inscrites dans le rapport «Les Langues de la France » de Bernard Cerquiglini (1999), les langues autochtones de Polynésie française et celles de Nouvelle-Calédonie sont désormais, aux yeux de l'État, des «langues de France ». Cette dénomination ne va cependant pas de soi dans un contexte ultramarin postcolonial ou en voie de décolonisation. Le processus de reconnaissance de ces langues s'inscrit dans une temporalité distincte de celle de l'Hexagone, selon l'histoire de chacune de ces deux collectivités dont la configuration linguistique, démographique et politique a induit des modalités différentes d'institutionnalisation des langues et cultures autochtones.

\section{Les langues autochtones dans le paysage linguistique contemporain}

Les langues autochtones de Nouvelle-Calédonie et celles de Polynésie française appartiennent au même groupe océanien de la famille austronésienne. La trentaine de langues autochtones que compte l'archipel calédonien, les «langues kanak $»^{1}$, sont issues d'une protolangue commune parlée par des navigateurs austronésiens arrivés dans l'archipel il y a environ 3000 ans. Seul le faga'uvea, d'une origine polynésienne plus récente, fait exception (Moyse-Faurie, Rivierre, Vernaudon 2012). Même si elles partagent un fonds généalogique océanien commun, les langues kanak sont très différentes les unes des autres. À titre d'exemple, la phonologie du nengone de l'ile de Maré dénombre 39 consonnes et 10 voyelles alors que le nrââ kwênyii de l'ile des Pins dispose de 25 consonnes et de 35 voyelles. La multiplicité des langues participait autrefois à un équilibre utile aux échanges matrimoniaux entre groupes exogames et patrilocaux. Les femmes, qui venaient résider dans le clan de leur époux, étaient encouragées à parler leur langue d'origine à leurs enfants afin de maintenir les liens avec le clan utérin. Le plurilinguisme des individus, parlant a minima la langue de leur clan paternel et celle de leur clan utérin, permettait la communication entre les différents ensembles

\footnotetext{
${ }^{1}$ Le mot « kanak » est une réappropriation de l'appellation coloniale canaque, laquelle vient du hawaiien kanaka « être humain ». Son usage s'est diffusé au XIX ${ }^{\mathrm{e}}$ siècle dans le vocabulaire marin puis colonial pour désigner les populations autochtones par opposition aux équipages puis aux colons. Il n'y avait pas d'ethnonyme générique antérieur à la colonisation pour désigner les premiers habitants de la Nouvelle-Calédonie.
} 
ethnolinguistiques de 1'archipel. Selon Maurice Leenhardt (1946: XVI), « la possession de plusieurs langues [était] l'un des éléments essentiels de la culture personnelle de l'ancien Canaque (sic) ».

Les langues de Polynésie française, appelées « langues polynésiennes » ou « reo mā'ohi ${ }^{2}$ », sont issues du proto polynésien centro-oriental et correspondent à une étape du peuplement austronésien beaucoup plus récente, il y a environ 1000 ans. Elles présentent un état de diversification beaucoup moins prononcé qu'en Nouvelle-Calédonie et la connaissance de l'une d'elles donne un accès relativement facile aux autres. Soulignant l'imprécision du critère de compréhension mutuelle sur lequel repose la distinction entre langues et dialectes ${ }^{3}$, Jean-Michel Charpentier et Alexandre François $(2015: 22)$ indiquent prudemment que « le nombre exact de "langues différentes" parlées traditionnellement en Polynésie française oscille entre cinq et huit ».

Contrairement à la situation calédonienne pré-occidentale, il n'y avait probablement pas de plurilinguisme individuel marqué dans l'ensemble géographique qui correspond aujourd'hui à la Polynésie française car, si l'on excepte les échanges entre archipels où la proximité des langues permettaient une relative intercompréhension, les habitants de chaque archipel vivaient dans des environnements linguistiques homogènes.

À ce paysage linguistique autochtone se sont ajoutés progressivement, à partir de la seconde moitié du XIX ${ }^{\mathrm{e}}$ siècle, le français, langue de colonisation, et toutes les langues des migrations de pays voisins et de pays plus lointains. Le français est désormais la seule langue officielle, langue véhiculaire et principale langue de scolarisation dans les deux collectivités. Des regroupements de population kanak autour de la mission de Saint Louis, près de Nouméa, au cours de la seconde moitié du $\mathrm{XIX}^{\mathrm{e}}$ siècle, sont à l'origine de l'émergence d'un créole à base lexicale française, le tayo, qui n'a cependant pas connu l'expansion des trois autres créoles de la région, à base lexicale anglaise, le bislama du Vanuatu, le pijin des iles Salomon et le tok pisin de Papouasie-Nouvelle-Guinée (Ehrhart 2012).

Le Tableau 1 présente les principales langues en présence et leurs statuts dans les deux collectivités.

\begin{tabular}{|c|c|c|}
\cline { 2 - 3 } \multicolumn{1}{c|}{ Nombre } & Nouvelle-Calédonie & $\begin{array}{c}\text { Polynésie } \\
\text { française }\end{array}$ \\
\hline Langues parlées & $\sim 40$ & $\sim 12$ \\
\hline Langue officielle & français & français \\
\hline Langues autochtones & 28 langues kanak & $\sim 7$ langues polynésiennes \\
\hline Créole local & Tayo & - \\
\hline $\begin{array}{c}\text { Langues issues de migrations } \\
\text { d'Océanie }\end{array}$ & $\begin{array}{c}\text { wallisien, futunien, } \\
\text { tahitien, langues } \\
\text { vernaculaires du Vanuatu } \\
\text { et bislama... }\end{array}$ & $\begin{array}{c}\text { wallisien, fidjien... } \\
\text { Langues issues de migrations plus } \\
\text { lointaines }\end{array}$ \\
$\begin{array}{c}\text { vietnamien, javanais, } \\
\text { mandarin, cantonais, } \\
\text { japonais, anglais... }\end{array}$ & $\begin{array}{c}\text { hakka, autres langues } \\
\text { chinoises, anglais... }\end{array}$ \\
\hline
\end{tabular}

\footnotetext{
${ }^{2}$ Pour plus de précision sur l'emploi du terme tahitien $m a \bar{a}$ 'ohi et sur sa charge idéologique et politique, cf. Saura, 2008.

${ }^{3}$ Deux idiomes sont considérés comme des dialectes d'une même langue tant qu'il y a intercompréhension entre eux.
} 
Tableau 1 - Multilinguisme sociétal et statuts des langues

À l'occasion du recensement de 2014, environ 67500 habitants de Nouvelle-Calédonie de 15 ans et plus déclaraient parler une langue kanak, soit $33 \%$ des personnes de cette tranche d'âge, toutes ethnies confondues, et soit quatre Kanak sur cinq (ISEE-NC 2014). Douze des 29 langues kanak (avec le tayo) comptent moins de mille locuteurs. Le drehu et le nengone, respectivement langues de Lifou et de Maré aux iles Loyauté, sont les deux langues kanak les plus parlées (16 000 et 9000 locuteurs), y compris dans le Grand Nouméa où les locuteurs de ces deux langues sont désormais plus nombreux que dans leur aire linguistique d'origine.

En Polynésie française, 152870 personnes de 15 ans et plus déclaraient, en 2017, comprendre, parler, lire et écrire une langue polynésienne, soit $71 \%$ de cette tranche d'âge (ISPF 2017) ${ }^{4}$. L'usage du tahitien est largement plus répandu que celui des autres langues polynésiennes.

Le français est la langue dont la connaissance est la mieux partagée dans les deux collectivités, même si une enquête conduite en 2013 en Nouvelle-Calédonie révèle "qu'un quart des Calédoniens âgés de 16 à 65 ans éprouve des difficultés face à l'écrit en français » (Benoit, Denis 2013: 1). Nous ne disposons pas de données équivalentes en Polynésie française, mais le recensement de 2017 indique que $96 \%$ de la population polynésienne âgée de 15 ans et plus déclarent comprendre, parler, lire et écrire le français (181929 personnes) (ISPF 2017).

En Nouvelle-Calédonie, aucune des langues kanak n'est véhiculaire, alors que le tahitien est pratiqué dans l'ensemble de la Polynésie française, conjointement au français. Jean-Michel Charpentier et Alexandre François $(2015: 49)$ détaillent à ce propos la situation de « triglossie » polynésienne contemporaine où, pour des raisons historiques et politiques, le français est dominant dans l'administration et le système éducatif, par rapport aux langues polynésiennes, mais où le tahitien domine à son tour très largement les autres langues polynésiennes. Pour acquérir sa fonction véhiculaire, le tahitien a bénéficié depuis le début du XIX ${ }^{\mathrm{e}}$ siècle de son statut de première langue d'évangélisation, du poids démographique des iles de la Société, puis de la concentration du pouvoir administratif, politique et économique sur l'ile de Tahiti.

\section{Bref historique de l'enseignement des langues}

Les langues autochtones ont été régulièrement menacées dans leur transmission depuis le contact avec l'Occident. La chute démographique des populations océaniennes amorcée à la fin du XVIII ${ }^{\mathrm{e}}$ siècle, à la suite des épidémies dont les navires occidentaux ont été les vecteurs (Rallu 1990), a lourdement affecté la diffusion entre générations des langues et des savoirs locaux issus de cultures à tradition orale. Certaines langues ont cependant bénéficié d'une attention soutenue comme langues d'évangélisation au cours du XIX ${ }^{\mathrm{e}}$ siècle. C'est particulièrement l'œuvre de la London Missionary Society, fondée en 1795, qui choisit Tahiti pour sa première implantation dans le Pacifique en 1797. La stratégie d'évangélisation protestante associait alors étroitement évangélisation, traduction et alphabétisation (Nicole 1988: 10). Un premier catéchisme fut publié en tahitien dès 1801 et la première édition complète de la Bible en tahitien parut en 1838. Outre leur travail de normalisation orthographique et de traduction, les missionnaires déployèrent une intense activité d'alphabétisation vernaculaire. En 1823, le commandant Duperrey écrivait dans son rapport au ministre de la Marine et des Colonies à

\footnotetext{
${ }^{4}$ Les populations totales de la Nouvelle-Calédonie et de la Polynésie française s'élèvent respectivement à 268767 (ISEE-NC, 2014) et 275918 habitants (ISPF, 2017).
} 
propos des écoles missionnaires : «Tous les naturels de Tahiti savent lire et écrire » (cité par Nicole $1988: 1$ ).

L'œuvre missionnaire protestante se poursuivit à l'ouest du Pacifique et arriva en NouvelleCalédonie. Après une première tentative infructueuse dans le sud de la Grande Terre et à l'ile des Pins, la London Missionary Society s'implanta durablement aux iles Loyauté à partir des années 1840. Un rapport du délégué L. de Salins, en 1885, adressé au gouverneur Le Bouchet signale à propos de l'ile de Maré que « toute la population qui n'a pas dépassé 27 ans sait lire et écrire le maréen. Il faut être de mauvaise foi pour nier les résultats obtenus par les pasteurs anglais » (cité par R. Leenhardt $1980: 123$, note 21 ).

L'œuvre missionnaire a conduit à une profonde acculturation judéo-chrétienne des sociétés océaniennes tout en participant dans le même mouvement à la pérennisation d'une poignée de langues autochtones choisies pour diffuser la nouvelle religion, leur conférant ainsi la fonction de langues d'enseignement, fonction plus tard contestée par le colonisateur.

\section{L'école coloniale et ses velléités d'imposition du français}

À travers les actes juridiques dont on trouve la trace dès le début de l'implantation coloniale, l'administration française manifesta son intention de franciser les populations locales. En Nouvelle-Calédonie, dix ans après la prise de possession de la Grande Terre, l'arrêté du 15 octobre 1863 dispose que dans les écoles privées «l'enseignement portera sur : L'instruction morale et religieuse, la lecture, l'écriture, les éléments de la langue française, le calcul et le système légal des poids et mesures » et que « l'étude des idiomes calédoniens est formellement interdite dans toutes les écoles ». Le gouverneur Guillain explicite dans son arrêté l'objectif de ces dispositions : il s'agit de « faciliter nos relations avec les indigènes et l'accomplissement de notre mission civilisatrice à leur égard, ce qui exige, avant tout, qu'ils nous comprennent $»$. L'arrêté du 3 août 1905 précisant le fonctionnement des écoles primaires réaffirma à son article 12 que « le français sera seul en usage à l'école».

En Polynésie orientale, l'ordonnance du 30 octobre 1862, dix ans après la mise en place du protectorat sur le royaume de Pomare, rend obligatoire l'enseignement de la langue française dans les écoles de districts, au même titre que celui de la langue tahitienne. Stéphane Argentin et Alain Moyrand (2013:314, note 7) précisent à ce sujet :

Cette ordonnance instaure certes un enseignement des deux langues, mais son objectif est clairement mentionné par les termes utilisés dans un considérant : " que de tous les moyens employés pour hâter le développement de la civilisation parmi les populations indigènes, il n'en est pas de plus efficace que la propagation de la langue française ».

Après l'annexion du royaume de Pomare et l'instauration des Établissements français de l'Océanie (EFO) en 1880, un arrêté du 27 octobre 1897 rend obligatoire l'enseignement primaire dans toute l'étendue des EFO et précise à cet effet dans un considérant que « jusqu'ici, l'indifférence de la population indigène a été un réel obstacle à la diffusion de la langue française dans notre possession et qu'il importe de remédier au plus tôt à cet état de choses regrettable » (cité par Argentin, Moyrand 2013 : 314-315).

Les recherches archivistiques minutieuses de Marie Salaün $(2005$; 2015) en NouvelleCalédonie et en Polynésie française ont révélé combien ces velléités de francisation furent peu suivies d'effet sur le terrain. Les populations autochtones, qui vivaient principalement en autosubsistance des ressources horticoles et halieutiques, restaient indifférentes au français dont l'apprentissage ne fournissait à cette époque aucune plus-value socio-économique saillante. En outre, les moyens matériels et surtout humains déployés par l'administration coloniale pour instruire les enfants kanak ou polynésiens en langue française demeuraient très insuffisants. 
Dans un rapport publié en 1914, l'inspecteur Revel témoigne de la faible pénétration du français dans la société tahitienne du début du XIX ${ }^{\mathrm{e}}$ siècle :

Tout Français débarquant pour la première fois à Papeete est fort surpris d'entendre indigènes et européens s'exprimer en différents idiomes, dans lesquels dominent la langue maorie et la langue anglaise. L'usage de l'une et de l'autre est constant, même dans les familles métisses franco-tahitiennes, ou (sic) il n'est pas rare qu'une phrase, commencée en maorie se poursuivre en anglais parsemé d'expressions françaises. [...] Au chef-lieu, qui ne compte cependant que 1100 indigènes sur une population de 4000 personnes, les tahitiens parlent exclusivement leur langue et n'utilisent le français qu'à regret. En dehors de Papeete, les adultes ne comprennent en général pas le français. Dans les écoles, quelques-uns des élèves les plus anciens savent quelques mots, mais sont incapables de suivre une conversation. (cité par Salaün 2015 : 42-43)

C'est après la Seconde Guerre mondiale que l'idéologie du «tout français » finit par s'imposer dans les faits. L'accès progressif à la pleine citoyenneté des populations locales s'accompagne d'une massification de l'enseignement. L'État s'emploie à rattraper l'écart avec le modèle métropolitain en termes d'équipement, de formations des enseignants et de contenus d'enseignement. Dans un témoignage qui concerne la Polynésie française, mais qui pourrait s'appliquer tout aussi bien à la Nouvelle-Calédonie, Henri Lavondès rend compte du monopole du français à l'école et dans l'administration à la fin des années 1960 :

C'est l'enseignement à tous les niveaux (primaire et secondaire) et selon toutes ses modalités (enseignement public et enseignement privé) qui est l'instrument principal de la politique de francisation. Dès le niveau le plus élémentaire, la totalité de l'enseignement est dispensée en français. À aucun stade, dans l'enseignement public, le tahitien n'est enseigné. L'usage des langues vernaculaires est interdit dans le cadre des écoles, non seulement aux maitres, mais encore aux élèves qui ne sont pas autorisés à les employer pendant la classe et même dans leurs jeux pendant les récréations. Cela a pour but de faire acquérir aux élèves une connaissance pratique de la langue française et d'éviter qu'elle ne soit pour eux qu'une langue morte, sans utilisation possible dans le cadre de la vie quotidienne. En dehors de l'école, c'est encore le français qui occupe une position dominante. Les dialectes polynésiens sont tacitement ignorés dans tous les cas où la nécessité de communiquer ne rend pas strictement indispensable un recours au vernaculaire. Le français est la langue officielle de tous les services officiels $d u$ Territoire : administration, services techniques, justice, police... (Lavondès 1972 : 55)

La francisation fut généralement accompagnée de pratiques humiliantes, parfois violentes, pour les élèves locuteurs «natifs » d'autres langues que le français et il faut attendre la fin des années 1970 en Polynésie française et les années 1980 en Nouvelle-Calédonie pour que l'État infléchisse sa politique linguistique. Cette réorientation répond aux revendications identitaires locales qui émergent à partir de la fin des années 1960 et dont l'éducation devient un thème majeur. Les premières récriminations autour de l'école en Nouvelle-Calédonie sont alors formulées dans un contexte où, contrairement aux attentes, la massification de l'enseignement ne s'est pas accompagnée d'une promotion scolaire et sociale des Kanak :

Alors qu'il leur est théoriquement possible de se présenter aux examens longtemps réservés aux seuls Blancs, la part des diplômés dans la population kanak reste infime. Un vaste processus d'élimination fait d'abandons et de relégation vers 
les voies professionnelles les moins valorisées fait qu'à la fin des années 1970, si les enfants kanak représentent $55 \%$ des effectifs du Primaire, ils ne représentent plus que $20 \%$ des effectifs de la classe de fin de Secondaire, et $10 \%$ des reçus au titre qui permet l'accès aux études supérieures, le Baccalauréat (Kohler, Wacquant 1985). Cent vingt ans après la prise de possession par la France de la NouvelleCalédonie, neuf Mélanésiens sur dix n’ont donc aucun diplôme. (Salaün, Vernaudon 2009 : 69)

En Polynésie française, outre le discours identitaire $m \bar{a}$ 'ohi émergent, s'exprime aussi une préoccupation en termes de réussite scolaire :

Vers les années 1970, les membres de l'Assemblée territoriale interpellaient alors le système éducatif sur son incapacité à répondre aux besoins des élèves polynésiens et à tenir compte de leur vécu culturel. [...] Les résultats scolaires en 1971 montrent que dès l'enseignement primaire, plus d'un enfant sur quatre, au $C P$, ont deux ans de retard par rapport aux normes métropolitaines, et près de deux sur trois, en CM2, contre un sur trois en métropole. (Paia 2014 : 412)

Dans les deux contextes, prenant le contre-pied de la politique d'assimilation conduite jusque-là, des demandes d'adaptation de l'école aux réalités linguistiques et culturelles locales sont alors formulées comme remédiation à l'échec scolaire des élèves autochtones. Il a fallu, selon Marie Salaün (2013:60-61), que trois conditions soient réunies pour en arriver là : que soient effectivement mises en œuvre, à partir des années 1950, l'homogénéisation et la massification du système éducatif, sur le modèle national, prônant l'égalité des chances conjointement au "tout français »; ensuite, que les espoirs placés dans cette école soient au moins partiellement déçus et que le constat d'un échec scolaire soit dressé dans un contexte postcolonial (mais pas forcément décolonisé) où « la relégation des autochtones [était] devenue illégitime » alors qu'elle semblait aller de soi en contexte colonial; enfin, que l'école apparaisse, de manière plus ou moins vive selon les collectivités et les acteurs sociaux, comme un élément étranger aux sociétés autochtones et susceptible de les pervertir. Le témoignage de Marie-Adèle Néchérö-Jorédié, recueilli en 1987 durant les «Événements ${ }^{5}$ » alors qu'elle animait une école populaire kanak à Canala (cf. infra), est emblématique de ce ressenti :

Que sont devenus, seize ans après, les enfants de Canala entrés à l'école en 1960 ? On a constaté que Canala ne comptait qu'un bachelier (moi-même, je ne suis pas bachelière, je suis arrivée au niveau Bac). Autre constat : l'échec total de la réinsertion du petit Kanak dans son milieu, et l'échec pour appréhender les choses du monde moderne [...]. Il y avait aussi un déracinement affectif. L'enfant revenait chez lui, il ne savait pas parler sa langue. Moi, j'en parle parce que je suis restée dans cette école et que j'ai parlé français pendant des années et des années. Je suis branchée aujourd'hui sur l'école parce qu'il y a des choses dont j'ai souffert, des choses qui m'ont manquées. [...] Quand nous avons parlé de rupture avec le système éducatif actuel, c'était pour dire qu'il fallait qu'on se retrouve nous, chez nous, dans notre langue, avec notre vie. (Néchérö-Jorédié 1988 : 246-254)

La première demande officielle d'une prise en compte de la spécificité kanak dans l'enseignement néo-calédonien date de 1971, lorsque des élus locaux réclamèrent l'application au territoire de la loi Deixonne ${ }^{6}$, qui régissait en Métropole depuis 1951 l'enseignement des langues régionales de France. Consulté sur la recevabilité de la demande, le vice-recteur en

\footnotetext{
${ }^{5}$ C'est ainsi que l'on nomme pudiquement les quatre années de quasi guerre civile qui ont embrasé la NouvelleCalédonie à partir de 1984.

${ }^{6}$ Loi n ${ }^{\circ}$ 51-46 du 11 janvier 1951 relative à l'enseignement des langues et dialectes locaux.
} 
poste à Nouméa répondit en 1975 que la pratique de la langue française devait être «constante » à l'école primaire et que «la présence de langues vernaculaires mélanésiennes parmi les épreuves de baccalauréat ne saurait, à brève ou moyenne échéance, être valablement envisagée » (cité par Salaün, Vernaudon 2011 : 136). Face à ce refus, la question de la place des langues kanak et du français à l'école fut rapidement investie d'une dimension politique. En 1984, une des premières décisions de la majorité territoriale nouvellement élue et dirigée par Jean-Marie Tjibaou fut d'abroger l'ensemble des dispositions héritées de la colonisation interdisant l'usage des langues kanak à l'école et dans les publications ${ }^{7}$. Un an plus tard, les indépendantistes lancèrent un mot d'ordre de boycott des écoles "coloniales". On vit la création, éphémère, d'écoles communautaires, les écoles populaires kanak (EPK) où l'enseignement se faisait en langues locales (Néchérö-Jorédié 1988 ; Gauthier 1996), alors que dans le camp loyaliste, la revendication de reconnaissance des langues kanak fut assimilée à une contestation de la présence de la France et de ses ressortissants, voire à une menace pour l'intégrité de la République. À l'issue des «Événements », le consensus autour de la nécessité d'un retour à la paix civile, exprimé par la signature des accords de Matignon-Oudinot en 1988, impliquait une réponse des institutions aux revendications culturelles des nationalistes kanak. Les provinces ${ }^{8}$ obtinrent la possibilité de procéder à l'adaptation des programmes en fonction de leurs réalités culturelles et linguistiques dans le premier degré avec un quota de cinq heures hebdomadaires. La loi Deixonne fut étendue à la Nouvelle-Calédonie et quatre langues kanak (drehu, nengone, ajië, paicî) furent introduites dans les épreuves du baccalauréat en 1992. L'accord de Nouméa, signé en 1998 et toujours en vigueur au moment où nous écrivons ces lignes, a ouvert des perspectives de réforme éducative plus ambitieuses encore en prévoyant le transfert progressif des compétences de l'enseignement primaire et secondaire. Il dispose que « les langues kanak sont, avec le français, des langues d'enseignement et de culture ». En 1999, une filière de Langues et Cultures Régionales (LCR) fut mise sur pied à l'Université de la Nouvelle-Calédonie. Depuis la rentrée 2006, les programmes calédoniens de l'école primaire prévoient un enseignement facultatif des langues kanak à raison de 7 heures en maternelle et de 5 heures à l'école élémentaire «pour les enfants dont les parents en ont exprimé le vœu » ${ }^{9}$. Il n'y a pas de liste restrictive des langues kanak enseignées. Ces dernières sont proposées en fonction des ressources mobilisables par les provinces et de la demande des parents. Un concours de professeur des écoles «spécial » a été créé en 2006 pour favoriser le recrutement d'enseignants bilingues français/langues kanak.

En Polynésie française, le processus de reconnaissance des langues autochtones débuté dans les années 1970 fut davantage consensuel :

En 1975, le Conseil du Gouvernement demande la création d'une commission pour analyser la possibilité d'introduire la langue tahitienne à l'école. Après avoir évalué le système éducatif, la commission conclut qu'une bonne connaissance de la langue maternelle est profitable à l'enseignement du français et préconise l'enseignement progressif du tahitien à l'école primaire optionnel au second degré et un programme de formation obligatoire des enseignants. (Paia 2014 : 413)

Le statut d'autonomie de gestion accordé à la Polynésie française le 12 juillet 1977 conféra au gouvernement local une compétence en matière d'enseignement des langues locales et «à défaut de consensus sur la question de l'indépendance (vers laquelle l'autonomie constitu[ait] pour certains une étape, pour d'autres un rempart), un accord implicite [s'établit] autour de la

\footnotetext{
${ }^{7}$ Délibération $\mathrm{n}^{\circ} 333$ de la commission permanente de l’Assemblée territoriale du 29 février 1984.

${ }^{8}$ La Nouvelle-Calédonie a été divisée en 1989 en trois provinces : sud, nord et iles Loyauté, les deux dernières étant de majorité indépendantiste depuis cette date.

${ }^{9}$ Délibération $\mathrm{n}^{\circ} 118$ du 26 septembre 2005 portant programmes de l'école publique de la Nouvelle-Calédonie et délibération $n^{\circ} 381$ du 10 janvier 2019 portant organisation de l'enseignement primaire de la Nouvelle-Calédonie.
} 
promotion de la culture polynésienne, très vite rebaptisée culture $m \bar{a}^{\prime} o h i$ » (Saura $2008: 129$ ). En 1980, le conseil de gouvernement prit la décision, rendue exécutoire par le haut-commissaire de la République, de donner à la langue tahitienne qualité de langue officielle du territoire de la Polynésie française, conjointement avec la langue française ${ }^{10}$. La modification de l'article 2 de la Constitution française en 1992 (cf. infra) entrainera néanmoins l'invalidation, pour le tahitien, de sa qualité de langue officielle à partir de la loi organique de 1996 et dans tous les statuts ultérieurs. La loi Deixonne fut étendue à la Polynésie française en 1981 et l'enseignement du tahitien fut introduit progressivement en maternelle et au primaire ( 2 heures 40 minutes par semaine) et dans le premier cycle du secondaire en tant que langue vivante optionnelle. Une épreuve facultative de tahitien fut instaurée au baccalauréat en 1981 puis aux examens du brevet d'études professionnelles et du certificat d'aptitude professionnelle en 1991. Une filière universitaire de reo mā'ohi ouvrit en 1993 ainsi qu'une section tahitien-français au concours des enseignants du second degré en 1997. Si le tahitien est très largement favorisé juridiquement, la diversité linguistique interne n'est pas complètement sacrifiée, puisque la loi organique de la Polynésie française de 1984 et les versions ultérieures prévoient que, sur décision de l'assemblée territoriale, la langue tahitienne peut être remplacée dans certaines écoles maternelles et primaires par l'une des autres langues polynésiennes.

\section{Convergences et divergences}

Après ce bref rappel des avancées statutaires des langues kanak et polynésiennes, abordons à présent les convergences et divergences significatives de ce processus selon les deux collectivités.

Précisons d'abord leur statut juridique puisqu'il détermine la répartition des compétences entre les gouvernements locaux et le gouvernement national, en particulier en matière de pilotage des politiques culturelles et éducatives.

La Polynésie française est un pays d'outre-mer au sein de la République, dont l'autonomie est régie par l'article 74 de la Constitution française. L'article 1 de sa loi organique $n^{\circ} 2004-$ 192 du 27 février 2004 dispose :

La Polynésie française se gouverne librement et démocratiquement, par ses représentants élus et par la voie du référendum local, dans les conditions prévues par la présente loi organique.

La République garantit l'autonomie de la Polynésie française; elle favorise l'évolution de cette autonomie, de manière à conduire durablement la Polynésie française au développement économique, social et culturel, dans le respect de ses intérêts propres, de ses spécificités géographiques et de l'identité de sa population.

L'assemblée de la Polynésie française, élue au suffrage universel direct, règle par ses délibérations les affaires de la collectivité. Cette assemblée élit, en son sein, le président du gouvernement de la Polynésie française.

La Nouvelle-Calédonie est une collectivité sui generis qui se subdivise en trois provinces et dont le statut transitoire est régi par le titre III de la Constitution française. La loi organique n 99-209 du 19 mars 1999 définit ses institutions actuelles et les modalités de transfert de compétences non régaliennes, dont celles de la culture et de l'enseignement. Les assemblées de provinces, réunies en congrès, élisent le gouvernement de la collectivité.

Ces statuts confèrent à la Polynésie française et à la Nouvelle-Calédonie une autonomie relative en matière de politique linguistique, strictement encadrée néanmoins par la Constitution

\footnotetext{
${ }^{10}$ Décision n ${ }^{\circ}$ 2036/VP du 28 novembre 1980.
} 
française qui dispose en son article 2, suite à sa révision du 25 juin 1992, que « la langue de la République est le français ». Par un arrêt du 13 juin 2013, le Conseil d'État, par exemple, déclarait illégales deux lois adoptées par l'assemblée de la Polynésie française au motif que plusieurs orateurs s'étaient exprimés en tahitien lors des débats. Le système éducatif des deux collectivités présente par ailleurs une forte homologie avec le modèle national, pour la structuration du cursus, les contenus d'enseignement et la sélection/formation des enseignants. Le socle national commun des connaissances et des compétences s'y applique ${ }^{11}$ et les programmes locaux sont une copie à peine retouchée des programmes de la métropole.

Dans aucune des deux collectivités ne se sont déployées durablement des écoles spécifiques sous contrat, dédiées à la revitalisation des langues locales, sur le modèle des écoles Diwan en Bretagne par exemple. Même s'il existe des initiatives associatives en dehors du temps scolaire, la question de l'enseignement des langues locales reste débattue prioritairement dans le cadre de l'enseignement ordinaire.

\section{Consensus politique et adhésion populaire}

On a vu plus haut le vif contraste entre le contexte polynésien, relativement consensuel sur l'introduction du tahitien à l'école, et le contexte néo-calédonien, où la question de la promotion de la culture et des langues kanak est restée conflictuelle jusqu'aux accords de Matignon de 1988. La composition démographique de deux sociétés y joue pour beaucoup. Contrairement à la Nouvelle-Calédonie où les Kanak sont minoritaires, soit $39,1 \%$ de la population totale (ISEE-NC 2014), la Polynésie française n'a pas été une colonie de peuplement et la proportion des habitants ayant une ascendance autochtone, même partielle, y reste largement majoritaire. En conséquence, et comme le confirme l'enquête de Marie Salaün (2011 : 141) auprès des autorités politiques et pédagogiques, des équipes éducatives et des familles, il semble admis qu'enseigner les langues et culture polynésiennes " relève de l'intérêt général », conviction qui transcende les appartenances politiques ou les orientations idéologiques des uns et des autres. La charte de l'Éducation, votée à l'unanimité par les élus de l'Assemblée de la Polynésie française le 29 août 2011, dispose d'ailleurs que :

L'objectif de l'École est la réussite de tous les élèves. Cette réussite impose la maitrise du langage qui passe par le développement des compétences linguistiques en français, en langues polynésiennes et en langues étrangères. L'École doit tirer profit de la diversité linguistique de la société polynésienne pour favoriser le plurilinguisme tout au long de la scolarité.

Le processus de reconnaissance des langues kanak à l'école fut au contraire l'objet de vives tensions, au moins jusqu'à la période des « accords », entre indépendantistes et loyalistes en Nouvelle-Calédonie. Dans son article au titre révélateur «Les langues kanak ont-elles manqué leur (r)entrée à l'école calédonienne ? », Véronique Fillol observe :

Le contexte actuel, à savoir le transfert de compétences du second degré (2012) mais aussi le Grand débat (2010) ont bien évidemment ravivé des (op)positions politiques: nécessaire adaptation du système scolaire pour les uns (indépendantistes), rattachement au modèle national pour les autres (loyalistes) et (op)positions idéologiques (monolinguisme vs plurilinguisme(s)), avec bien entendu des configurations bien plus complexes selon les contextes et les moments. (Fillol 2013 : 52)

11 Cf. par exemple pour la Nouvelle-Calédonie : https://denc.gouv.nc/sites/default/files/documents/ socle_commun_connaissances.pdf. Et pour la Polynésie française : http://monvr.pf/socle-commun. 
Ces clivages se prolongent dans la segmentation provinciale de la Nouvelle-Calédonie. Voulue par le législateur pour permettre aux indépendantistes kanak d'accéder au pouvoir dans les exécutifs provinciaux du Nord et des Iles, elle débouche sur la distribution de compétences éducatives entre la collectivité Nouvelle-Calédonie et ses trois provinces ${ }^{12}$, ce qui complexifie le pilotage de l'enseignement en général, et celui de l'enseignement des langues kanak en particulier, et entrave souvent les synergies à l'échelle du pays. Pour compenser cette fragmentation, un service de l'enseignement des langues et de la culture kanak (SELCK) ${ }^{13} \mathrm{a}$ été créé en 2012. Il est chargé d'animer, de contrôler et d'évaluer les actions relatives à l'enseignement des langues kanak dans les écoles primaires et dans les établissements d'enseignement du second degré publics et privés. Mais au vu des difficultés rencontrées, la tentation est grande de réduire l'enseignement des langues et de la culture kanak à un enseignement de la culture kanak... en français.

\section{Enseignement facultatif ou obligatoire}

En raison de l'article 2 de la Constitution française évoqué plus haut, l'enseignement des langues locales ne "saurait revêtir, au nom du principe d'égalité, un caractère obligatoire pour les élèves » (Argentin, Moyrand 2014 : 321). Les programmes calédoniens n'offrent un enseignement de langue kanak qu'aux enfants «dont les parents en ont exprimé le vœu » ${ }^{14}$. Dans les faits cependant, en province des iles, où la population est presque exclusivement kanak, tous les élèves suivent, selon des volumes et des modalités variables, un enseignement de/en langues kanak. En provinces Nord et Sud, le principe de l'inscription volontaire est appliqué et les élèves inscrits sont sortis de leur classe principale pour participer à l'enseignement de/en langue kanak, ce qui pose un certain nombre de contraintes organisationnelles. Véronique Fillol, sur la base de ses enquêtes sociolinguistiques, résume de manière lapidaire l'état d'esprit dominant :

Le discours que nous synthétisons est le suivant : "OUI pour l'enseignement des langues kanak (bien que nombreux soient sceptiques quant aux effets sur les résultats scolaires) mais uniquement pour les Kanak». (Fillol 2013 : 59)

La situation est très différente en Polynésie française où l'enseignement des langues polynésiennes est, comme ailleurs sur le territoire national, facultatif en droit constitutionnel, « mais au regard du droit positif de la Collectivité, dans le premier degré, il est obligatoire et semble satisfaire la Polynésie sans déranger l'État» (Argentin, Moyrand 2014 : 324). Les programmes scolaires prévoient que tous les élèves, sans distinction d'origine et sans accord préalable de leurs parents, fassent $2 \mathrm{~h} 30$ de tahitien (ou d'une autre langue polynésienne) par semaine.

\section{Les langues dans les projets nationalistes kanak et mā'ohi}

Si les partis indépendantistes kanak et $m \bar{a}$ 'ohi revendiquent la reconnaissance des langues autochtones comme langues d'enseignement, il s'en faut de beaucoup pour que cette revendication s'inscrive dans un projet global équivalent. En Nouvelle-Calédonie, les élus indépendantistes ont pris acte du rôle véhiculaire de la langue française et aucune des 28 langues

\footnotetext{
12 Il y a trois directions provinciales de l'enseignement et une direction de l'enseignement de la NouvelleCalédonie. À titre de comparaison, il n'y a qu'une direction de l'enseignement en Polynésie française, au sein de laquelle une cellule des langues et culture polynésiennes est chargée de piloter cet enseignement sur l'ensemble du territoire.

13 Arrêté n 2012-161/GNC du 9 janvier 2012.

14 Délibération $\mathrm{n}^{\circ} 381$ du 10 janvier 2019 portant organisation de l'enseignement primaire de la NouvelleCalédonie.
} 
kanak n'est pressentie pour s'y substituer comme langue officielle, ni même d'y être adjointe comme langue co-officielle. Dans le bimensuel du Front de Libération Nationale Kanak et Socialiste, La voie du FLNKS, ${ }^{\circ} 17$, de mars 2012, un projet de constitution de Kanaky est présenté. On peut y lire à l'article 1 du titre 1 « Principes fondamentaux » :

Le Peuple kanak constitue une communauté nationale et pluriethnique, libre, unie et souveraine, fondée sur la solidarité de ses divers éléments. [...]

La langue officielle [de Kanaky] est le français.

L'État [de Kanaky] reconnait et garantit l'usage des langues kanak.

Cette pleine intégration du français au projet indépendantiste kanak ne date pas d'hier puisqu'en 1987, Marie-Adèle Néchérö-Jorédié expliquait déjà à propos de l'école populaire kanak de Canala :

Nous n'avons jamais dit que nous ne voulions pas de cette langue française, mais nous avons refusé la manière dont on nous l'imposait, et si mal que les enfants ne parvenaient pas à la maitriser. Voilà. On pourrait me demander " pourquoi le français? ", alors que nous sommes en plein milieu anglophone. Parce qu'on ne peut pas enlever cent trente ans de présence française, et que de toute façon, cet anglais-là dans lequel on baigne tout le temps, on l'a mal appris, on ne le connait même pas. Donc tant qu'à faire, puisqu'il n'y a pas eu encore de choix politique de langue nationale, nous disons dans notre école : il y a une langue qui est là, qui est véhiculaire, qui est une langue de communication, utilisons-la mais en cherchant les bons outils pour l'appréhender. Et sur le plan politique, nous disons : "Quand nous avons envie de nous battre avec quelqu'un, eh bien! il faut prendre ses propres outils pour se battre; et pour aller affronter le Français, eh bien! il faut maitriser sa langue pour pouvoir démonter ses arguments. " (Néchérö-Jorédié $1988: 254)$

Les indépendantistes $m \bar{a}$ 'ohi placent au contraire parmi leurs revendications politiques prioritaires la promotion de la langue tahitienne comme langue officielle. La voie défendue de longue date est celle d'une co-officialité avec la langue française. Si l'on fait abstraction de l'obstacle constitutionnel, divers facteurs rendent viable la promotion du tahitien comme langue co-officielle en Polynésie française. Le tahitien, langue d'évangélisation au-delà de l'archipel de la Société, est déjà véhiculaire. Par ailleurs, la proximité des langues polynésiennes entre elles favorise l'émergence d'un « néo-tahitien », koinè qui emprunte de nombreux vocables aux autres langues de Polynésie française dans l'usage quotidien, mais parfois aussi aux autres langues polynésiennes de la région. Ce processus favorise la déterritorialisation de la langue tahitienne, cette dernière étant de moins en moins perçue comme attachée à un archipel particulier - celui des iles de la Société -, mais comme un bien commun des Mā'ohi (Saura 2008). Le tahitien est aussi utilisé quotidiennement dans l'espace public et médiatique, entre autres par les hommes politiques et les journalistes, qui débattent, informent et commentent l'actualité locale, nationale et internationale, ce qui participe largement à sa décontextualisation (Salaün 2013). Le tahitien a également acquis une forte valeur d'échange dont atteste l'importance de sa maitrise dans les épreuves de certains concours administratifs.

\section{Des langues « de France»?}

L'appel à communication de ce numéro thématique rappelle que la conséquence la plus manifeste du Rapport Cerquiglini (1999) fut institutionnelle, la Délégation à la langue française 
ajoutant en 2001, la séquence « et aux langues de France » à son nom et « initiant une politique explicite d'ouverture et de soutien à la diversité linguistique ». À rebours du projet unilingue formulé par l'abbé Grégoire dans son rapport devant la Convention nationale du 4 juin 1794 qui corrélait l'universalisation de l'usage de la langue française à l'anéantissement des « patois » (Grégoire, $1794: 1$ ), l'association étroite des «langues de France » à la "langue française » dans le nom d'un organe gouvernemental interministériel encourage une réconciliation linguistique et consacre l'appartenance des langues régionales au patrimoine culturel de la France.

Ce positionnement symbolique, en principe favorable aussi aux langues kanak et polynésiennes, peut cependant être interprété localement comme contradictoire avec les aspirations d'émancipation politique. Les conquêtes institutionnelles favorables aux langues kanak et polynésiennes, dont les plus emblématiques sont probablement la promotion éphémère du tahitien comme langue officielle (1980-1996) et l'inscription des langues kanak dans l'accord de Nouméa (1998) comme «langues d'enseignement et de culture », relèvent d'initiatives autochtones, indépendantes de la trajectoire des langues régionales de l'Hexagone, et antérieures au Rapport Cerquiglini (1999). Les éléments historiques, sociologiques et politiques parcourus précédemment permettent de saisir pourquoi la dénomination « langues de France » utilisée par le gouvernement central, et applicable aux langues kanak et polynésiennes, peut paraitre incongrue du point de vue d'une portion non négligeable des habitants de la " périphérie » calédonienne et polynésienne. Elle revient à réaffirmer que, de jure, la NouvelleCalédonie ou la Polynésie française, c'est la France, principe en vertu duquel les langues de ces territoires sont la propriété de la France. Le Rapport Cerquiglini (1999 : 4) rappelle en effet les principes républicains français «qui tiennent que la langue, élément culturel, appartient au patrimoine national ; le corse n'est pas la propriété de la région de Corse, mais de la Nation » (c'est nous qui soulignons). On peut douter de l'adhésion unanime à ces principes par les communautés autochtones de Nouvelle-Calédonie et de Polynésie française. Ces deux collectivités sont inscrites sur la liste des territoires non autonomes des Nations Unies. Lors de la première des quatre consultations référendaires calédoniennes, le dimanche 4 novembre 2018, 43,3 \% des suffrages exprimés ont répondu « oui » à la question « Voulez-vous que la Nouvelle-Calédonie accède à la pleine souveraineté et devienne indépendante ? ». Le dimanche 6 mai 2018, le parti indépendantiste Tāvini Huira'atira a obtenu 23,11\% des voix pour les élections de 1'Assemblée de Polynésie française. Si les indépendantistes kanak ou mā'ohi considèrent désormais le français comme une composante souvent essentielle de leur répertoire langagier, ils ne se satisfont pas que leurs langues soient présentées comme une "propriété » de la nation française. La dénomination sonne aussi de façon paradoxale aux oreilles des loyalistes calédoniens qui ont longtemps considéré les langues kanak comme un étendard du nationalisme kanak opposé à la tutelle de la France. Les autonomistes polynésiens enfin ne veulent pas être en reste sur les questions d'affirmation identitaire face à leurs adversaires politiques indépendantistes. Édouard Fritch, actuel président autonomiste du gouvernement polynésien déclarait par exemple le 4 octobre 2016 devant la Quatrième Commission de l'Assemblée générale des Nations unis : «En tant qu'autonomistes, nous sommes parfaitement conscients que notre histoire n'est pas celle de la France. Notre mode de vie océanien et tropical n'est pas celui de la France européenne ». Régulièrement accusés par les indépendantistes d'être des ho'o 'äi'a, littéralement de vendre la patrie ( $\left.m \bar{a}^{\prime} o h i\right)$ à la France, ils n'ont pas l'audace de clamer que les langues polynésiennes sont des «langues de France » et propriété de cette dernière.

Pour autant, cette inscription a déjà offert et offre encore aux langues kanak et polynésiennes, en particulier via l'intervention de la DGLFLF, de nombreuses opportunités. À titre d'illustration, non exhaustive, la DGLFLF apporte un soutien financier régulier à l'Académie des langues kanak. Elle a subventionné plusieurs projets de publication sur ces langues, au 
format papier ou numérique ${ }^{15}$. Son Observatoire des pratiques linguistiques a financé en 2014 une enquête sur les représentations et les pratiques linguistiques des élèves de Tahiti (Salaün, Vernaudon, Paia 2016). Le bulletin d'information Langues et cité a consacré son $n^{\circ} 26$ aux langues kanak et son $\mathrm{n}^{\circ} 29$ aux langues polynésiennes. L'Atlas sonore des langues régionales de France, dont la DGLFLG est un des partenaires institutionnels, consacre une carte spécifique à chacune des deux aires, calédonienne et polynésienne ${ }^{16}$. Un ministre polynésien indépendantiste sollicitait même en 2012 le gouvernement central pour la création en Polynésie française d'une antenne de la DGLFLF, qui n'a cependant jamais vu le jour ${ }^{17}$. Ces initiatives appartiennent cependant principalement à l'espace académique et la diffusion des différents supports produits avec l'appui de la DGLFLF ne présage pas de la compréhension et de l'adhésion par les usagers locaux au message politique sous-jacent à la dénomination « langues de France ».

\section{Conclusion}

Des dynamiques locales débutées dans les années 1970 ont conféré aux langues kanak et polynésiennes, au sein de leurs contextes sociolinguistiques respectifs, une reconnaissance institutionnelle singulière et tout aussi significative que celle que leur apporte, au niveau national, leur inscription sur la liste des «langues de France ». Cette dernière dénomination n'est jamais utilisée dans l'espace public polynésien ou calédonien, car, par l'appropriation surplombante qu'elle dénote, elle parait contredire le processus de décolonisation dont la reconnaissance des langues autochtones est devenu un des symboles emblématiques. Les leviers politiques et financiers conférés par cette inscription, en particulier via l'intervention de la DGLFLF, profitent cependant aussi aux langues kanak et polynésiennes. Mais c'est au cœur même des sociétés et des institutions locales que se joue principalement l'avenir de ces langues. Malgré le plafond constitutionnel qui interdit leur reconnaissance comme langues officielles, le transfert à la Nouvelle-Calédonie et à la Polynésie française de larges compétences en matière de culture et d'enseignement permet d'envisager des dispositifs aux objectifs ambitieux pour leur promotion et leur transmission.

\section{Références}

Argentin S., Moyrand A., 2014, «Les langues polynésiennes au sein de l'école : entre malentendus linguistiques et crispations juridiques », in I. Nocus, J. Vernaudon, M. Paia (dir.), L'école plurilingue en Outre-mer: Apprendre plusieurs langues, plusieurs langues pour apprendre, Rennes, Presses universitaires de Rennes, pp. 313-326.

Benoit J., Denis T., 2013, «18\% des adultes en situation d'illettrisme », Synthèse, 29, pp.1-6. Cerquiglini B., 1999, Les langues de la France, Rapport au Ministre de l'Éducation nationale, de la Recherche et de la Technologie, et à la Ministre de la Culture et de la Communication, document consulté sur le site www.vie-publique.fr, le 29 octobre 2019. Charpentier J.-M., François A., 2015, Atlas linguistique de la Polynésie française, Berlin \& Papeete, Mouton de Gruyter \& Université de la Polynésie française.

\footnotetext{
${ }^{15}$ Cf. par exemple : Vernaudon, J., Bearune, S., Hmae, J., Mauberret, F., 2013, Nengone, Initiation à la langue de l'ile de Maré, Nouméa, UNC \& ALK, didacticiel en ligne. Lercari, C., Sam, L., Gowe, M., Vernaudon, J., 2001, Langue de Lifou, Qene drehu. Méthode d'initiation, Nouméa, UNC \& CDP-NC.

${ }^{16} \mathrm{Cf}$. https://atlas.limsi.fr/.

17 www.tahiti-infos.com/Promotion-des-langues-polynesiennes-Tauhiti-Nena-rencontre-Frederic-Mitterrand a43440.html, consulté le 03 novembre 2019
} 
Ehrhart S., 2012, L'Écologie des langues de contact. Le tayo, créole de Nouvelle-Calédonie, Paris, L'Harmattan.

Fillol V., 2013, « Les langues kanak ont-elles manqué leur (r)entrée à l'école calédonienne ?», in Becetti A., Blanchet P., Colonna R. (dir.), Politiques linguistiques et plurilinguismes. Du terrain à l'action glottopolitique, Paris : L'Harmattan, pp. 49-68.

FLNKS, 2012, " Projet de constitution de Kanaky », La voie du FLNKS, 17, mars 2012.

Gauthier, J., 1996, Les écoles populaires kanak. Une révolution pédagogique?, Paris, L'Harmattan.

ISEE-NC (Institut de la Statistique et des Études Économiques de Nouvelle-Calédonie), 2014, Recensement général de la population 2014, Nouméa, ISEE-NC.

ISPF (Institut de la Statistique de la Polynésie française), 2017, Recensement général de la population 2017, Papeete.

Kohler J.-M., Wacquant L., 1985, L'école inégale. Éléments pour une sociologie de l'école en Nouvelle-Calédonie, Nouméa, ORSTOM.

Lavondès H., 1972, " Problèmes sociolinguistiques et alphabétisation en Polynésie française », Cahiers de l'ORSTOM, série Sciences humaines, IX (1), pp. 49-61.

Leenhardt M., 1946, Langues et dialectes de l'Austro-Mélanésie, Paris, Institut d'ethnologie.

Leenhardt R., 1980, Au vent de la Grande Terre, Les Iles Loyalty de 1840 à 1895, Paris, Imprimerie Claude Bernard.

Moyse-Faurie C., Rivierre J.-C., Vernaudon J., 2012, « Les langues kanak », in Bonvallot J., Gay J.-C., Habert E. (dir.), Atlas de la Nouvelle-Calédonie, Nouméa, IRD, p. 26.

Néchérö-Jorédié M.-A., 1988, « Une école populaire kanake (EPK) : l'expérience de Canala Entretien avec Marie-Adèle Néchérö-Jorédié (juin 1987) », in Spencer, M., Ward, A., Connell, J. (dir.), Nouvelle-Calédonie, essais sur le nationalisme et la dépendance, Paris, L'Harmattan, 245-268.

Nicole J., 1988, Au pied de l'écriture, Histoire de la traduction de la Bible en tahitien, Papeete, Haere pō no Tahiti.

Paia M., 2014, «L'enseignement des langues et de la culture polynésiennes à l'école primaire en Polynésie française », in I. Nocus, J. Vernaudon, M. Paia (dir.), L'école plurilingue en Outre-mer: Apprendre plusieurs langues, plusieurs langues pour apprendre, Rennes, Presses universitaires de Rennes, pp. 409-429.

Rallu J.-L., 1990, Les Populations Océaniennes aux $19^{e}$ et $20^{e}$ Siècles, Paris, Institut national d'études démographiques \& Presses universitaires de France.

Salaün M., 2005, L'école indigène. Nouvelle-Calédonie. 1885-1945, Rennes, Presses universitaires de Rennes.

Salaün M., 2005b, Rapport d'évaluation de l'expérimentation «L'enseignement des langues et de la culture kanak à l'école primaire publique de la Nouvelle-Calédonie », Axe sociolinguistique, Paris, Université Paris 5 (multigraph.).

Salaün M., 2011, Renforcer l'enseignement des langues et cultures polynésiennes à l'école élémentaire. Contribution à l'évaluation de l'expérimentation ECOLPOM en Polynésie française : aspects sociolinguistiques, Rapport de recherche, ANR École Plurilingue Outre-mer, Paris, Université Paris 5 (multigraph.).

Salaün M., 2013, Décoloniser l'école? Hawaii, Nouvelle-Calédonie. Expériences contemporaines, Rennes, Presses Universitaires de Rennes.

Salaün M., 2015, « Les langues de l'école au temps des Établissements français de l'Océanie : ce que nous dit la législation coloniale, et ce qu'elle ne nous dit pas », Bulletin de la Société des Études Océaniennes, 336, pp. 24-53.

Salaün M., Paia M., Vernaudon J., 2015, «"Le tahitien, c'est pour dire bonjour et au revoir” : paroles d'enfants sur une langue autochtone en sursis », Enfances Familles Générations, 25 [revue en ligne], https://efg.revues.org/1156 
Salaün M., Vernaudon J., 2009, « La citoyenneté comme horizon : destin commun, demande sociale et décolonisation de l'École en Nouvelle-Calédonie aujourd'hui », Anthropologie et sociétés, 33 (2) : 63-80.

Salaün M., Vernaudon J., 2011, " "Les langues kanak sont, avec le français, des langues d'enseignement et de culture en Nouvelle-Calédonie", Et après ? », in Clairis, Ch., Costaouec, D. Coyos, J.-B., Jeannot-Fourcaud, B. (dir.), Langues et cultures régionales de France, Paris, L'Harmattan : 129-151.

Saura B., 2008, Tahiti Mā'ohi : culture, identité, religion et nationalisme en Polynésie française, Papeete, Au Vent des iles. 


\section{GLOTTOPOL}

Revue de sociolinguistique en ligne

Comité de rédaction : Michaël Abecassis, Salih Akin, Sophie Babault, Claude Caitucoli, Véronique Castellotti, Régine Delamotte, Robert Fournier, Stéphanie Galligani, Emmanuelle Huver, Normand Labrie, Foued Laroussi, Benoit Leblanc, Fabienne Leconte, Gudrun Ledegen, Danièle Moore, Clara Mortamet, Alioune Ndao, Isabelle Pierozak, Gisèle Prignitz.

Rédactrice en chef : Clara Mortamet.

Comité scientifique : Claudine Bavoux, Michel Beniamino, Jacqueline Billiez, Philippe Blanchet, Pierre Bouchard, Ahmed Boukous, Pierre Dumont, Jean-Michel Eloy, Françoise Gadet, Monica Heller, Caroline Juilliard, Jean-Marie Klinkenberg, Jean Le Dû (†), Marinette Matthey, Jacques Maurais, Marie-Louise Moreau, Robert Nicolaï, Didier de Robillard, Paul Siblot, Claude Truchot, Daniel Véronique.

\section{Comité de lecture pour ce numéro :}

Salih Akin, Carmen Alén Garabato, Sophie Babault, Philippe Blanchet, Henri Boyer, Véronique Castellotti, Marisa Cavalli, Jean-François De Pietro, Didier de Robillard, Alain Di Meglio, Ksenija Djordjevic, Jean Michel Eloy, Pascale Erahrt, Véronique Fillol, Monica Heller, Robert Fournier, Normand Labrie, Hervé Lieutard, Jean Le Dû (†), Marinette Matthey.

http://glottopol.univ-rouen.fr

ISSN : 1769-7425 